\title{
WECHAT VS. WHATSAPP: WHY CHINA IS NOT A NATION OF COPYCATS
}

\author{
Ghosia Ahmed and Miguel Baptista Nunes \\ School of Information Management, Sun Yat-Sen University, Guangzhou, China
}

\begin{abstract}
Whilst the preconception of China in the West is often that of a "copycat nation" or "the world's factory", the reality of China, however, is very different to how it had been portrayed to us. Coming to China from the West, it has become clear to us that China is establishing itself as a global leader in technology and innovation, far beyond Western nations. As a fast-paced and ever-evolving innovative nation, China already appears to be a leader in service innovation, cashless mobile payments, e-commerce and artificial intelligence. In particular, WeChat - the hi-tech social media platform that integrates functions for almost everything from messaging and calling services, cashless mobile payments, social media and e-commerce, to name a few - and the likes of which is difficult to find anywhere else in the world. WeChat is China's "app for everything"; it is the first app you will discover when you come to China.

In this paper, we compare WhatsApp and WeChat; two messenger apps, one from the East and the other from the West, that seemingly started with similar aims - to connect people through instant messaging and calling functions - yet the evolution of both has been so significantly different that it can be compared to the East-West dichotomy. Through this comparison and paper, we aim to draw attention to and provide a glimpse into the enormous realm of innovation and technological advancement in the world's most populous country.
\end{abstract}

\section{KEYWORDS}

Innovation, WhatsApp, WeChat, China, Social Media, Messenger Applications

\section{INTRODUCTION}

Growing up in the West, like most Westerners, we had various preconceptions about China derived from society, the media, the internet and stories from various people. Some of those preconceptions are misconceptions, particularly when it comes to the subject of originality and authenticity in technological advancement; our experience of living and working in China has taught us that China is no longer a "copycat nation" or "the world's factory", but instead it is becoming a global leader in technological and service innovation.

In this paper, we explore two of the leading and competing messenger applications, WhatsApp and WeChat, how their journeys started and how they have evolved respectively, followed by a comparison of their evolution and through which we draw attention to the huge contrast in that evolution. Through this comparison, we aim to not only show the contrast between two comparable applications - one, a global giant, and the other, a goliath in China, but also draw attention to the gap in understanding and reality of technological advancement between the West and China.

\section{BACKGROUND}

On $3^{\text {rd }}$ December 1992, Neil Papworth, an engineer at Vodafone, sent a message that changed the world: "Merry Christmas"; text messaging is now such an integral part of daily life for most people and often a preferred method of communication (McSweeney, 2018). Although text messaging initiated 27 years ago, in the past decade in particular, there has been a "massive adoption of a raft of mobile instant messenger applications and services on smartphones", often referred to as Over The Top (OTT) applications as they are independent of the 
mobile network (O’Hara et al, 2014: 1131). These include applications such as WhatsApp, WeChat, Facebook Messenger, iMessage, Viber, Skype, etc.

Digital technology revolution and the enormous growth in smart phone usage has led to over $66 \%$ of the world's population now using mobile phones and more than half are using social media, making mobile social networking (MSN) a key communication tool and a necessity in people's lifestyles (Wan et al, 2019). Further, the adoption of social media and messenger applications on smartphones is now in its billions, according to MessengerPeople (2019). O'Hara et al (2014: 1131) argue that whilst these applications are free or relatively cheap to use and they offer capabilities for communicating and sharing media, "What is curious about these applications is that, prima facie, they seem to offer little in the way of conceptual novelty; their cheapness eliding the fact that much of this functionality has been present on communication tools provided on PCs and Laptops for some time - in Gmail, Outlook, Skype.". The success of such applications may be attributed to not only the ubiquity of multiple communication methods and media, integration of various functions but to such applications being used as a key component of 'connection' in daily lives and situated "within the ebb and flow of lives lived together, within the web of other connections, both real and virtual" (O'Hara et al, 2014: 1143).

Whilst a wide range of messenger applications are available on the market, two of the leading, WhatsApp and its counterpart, WeChat - are smartphone messenger applications that were launched within two years of each other with the aim to provide reliable and free messaging and calling functionality to its users, both currently lead by comparison and have over 1 billion active monthly users each (MessengerPeople, 2019). Whilst the two applications initially started alike and with similar functionality and aims, the evolution and direction both have taken since has been extraordinarily different.

In the following sections we firstly explore the evolution and features of WhatsApp, followed by the evolution and features of WeChat and then make a comparison and discuss the similarities and differences between the two messenger applications to highlight their remarkably varying journeys.

\section{WHATSAPP EVOLUTION AND FEATURES}

WhatsApp was created in 2009 by Jan Koum and Brian Acton as an alternative to SMS messaging. Later, it was purchased by Facebook in 2014, although it is claimed that WhatsApp continues to operate as a separate application that focuses entirely on building a fast and reliable global messaging service (WhatsApp, 2020). The messenger application "is the pioneer and most popular leading messenger worldwide" with significant market penetration (Wan et al, 2019: 1094-1095). Further, the current 1.6 billion active monthly users in over 180 countries (WhatsApp, 2020; Statista, 2019), makes WhatsApp the leading messaging application globally in 169 countries (MessengerPeople, 2019).

WhatsApp is available on multiple platforms including Android, iPhone, Mac, Windows PC and Windows Phone. The application has evolved over the years with the primary focus still being on the messaging functionality but also incorporating calling functions. The application now supports the exchange of multimedia including text, photos, videos, documents, voice notes, voice and video calls, location sharing and 24-hour media status updates where users can share photos, text and videos with their chosen contacts. The application is still free to download and use. According to WhatsApp (2020), the application has been designed with user security and privacy at its core and claims to offer secure messaging with end-to-end encryption i.e. "Our messages and calls are secured with end-to-end encryption, meaning that no third-party including WhatsApp can read or listen to them."

In January 2018, WhatsApp launched its 'WhatsApp Business App' (Sulleyman, 2018). The application is separate from the main WhatsApp messenger application and like the messenger application, it is also free to download and use. The WhatsApp business application has been developed with the small business owner in mind where businesses can create catalogs for showcasing products and services, connecting with customers by using the inbuilt tools to automate, sort and quickly respond to messages (WhatsApp, 2020). In addition, the application also allows medium and large businesses to offer customer support and deliver notifications to their customers (WhatsApp, 2020). 


\section{WECHAT EVOLUTION AND FEATURES}

"Today, people can do almost everything with one device. But in China, you can do everything you want with one single app" (Charlotte et al, 2018: 1). WeChat, the first application one will most probably discover when arriving in China, also often referred to as China's "app for everything". WeChat, originally named and still referred to by most Chinese people as 'Weixin', was founded by the Chinese company Tencent in 2011 and is the main communication, social media networking, cashless payment and service application in the country. WeChat is available to users on multiple platforms including Android, iPhone, Mac, Windows PC and Windows Phone.

Although originally a messaging and calling application, WeChat has evolved in various ways since its launch. Wan et al (2019: 1094) describe the current version of WeChat as "A Way of Life", to emphasise the extent to which the application has evolved into an enormous multi-purpose platform targeted at consumers who seek daily life conveniences. The current platform of WeChat can be described as a multi-faceted portal where it offers services starting from messenger, communication and social media, to mini-programs and e-commerce to cashless mobile payment and business services - also known as WeChat Pay.

\subsection{WeChat Messenger}

The messenger side of WeChat offers text and voice messaging, group chats, audio calls and individual and group video calls, entertainment, social media and gaming (Wan et al, 2019). WeChat has also developed a feature called 'Moments' which allows users to share their experiences or news with WeChat friends who they have selected and granted permission to through photos, videos and web links, and receive 'likes' and comments as feedback on the posted content (Vodanovich et al, 2017). In addition, WeChat has also introduced novel features within a messenger application such as subscriptions, 'Split Bill', 'Red Packet' for sending money as a gift to family and friends, 'People Nearby' and 'Shake' which allow users to chat with other random users globally who are also shaking their phone simultaneously (Vodanovich et al, 2017).

\subsection{WeChat Pay}

By far the most notable part of WeChat and its evolution is called WeChat Pay (see Figure 1). "WeChat Pay empowers merchants to connect with consumers before, during, and after sales through innovative marketing platforms in the WeChat ecosystem" and is a leading payment platform used by Chinese consumers within and outside China and it is claimed to be used by over 800 million monthly active users (WeChat, 2020). In addition, licensed financial institutions are able to provide acquiring services to merchants as the partners of WeChat Pay, and merchants or store owners, whether online or offline, can sell products or services through WeChat Pay after integrating it and comprehensive support is offered to merchants to help grow their businesses and improve customer experience, utilise marketing resources and increase traffic (WeChat, 2020). WeChat Pay offers services including the following:

- Quick Pay -WeChat Pay payment code presented by user and scanned by merchant

- QR Code Payment - Users scan code, confirm the amount, and make the payment

- Mini Program Payment - Users make payments in Mini Programs inside WeChat

- Official Account Payment - Users pay inside a merchant's Official Account

- In-App Payment - Users make purchases in merchants' applications using WeChat Pay

- Web Payment - Users use WeChat to scan a payment code on a webpage to pay (WeChat, 2020)
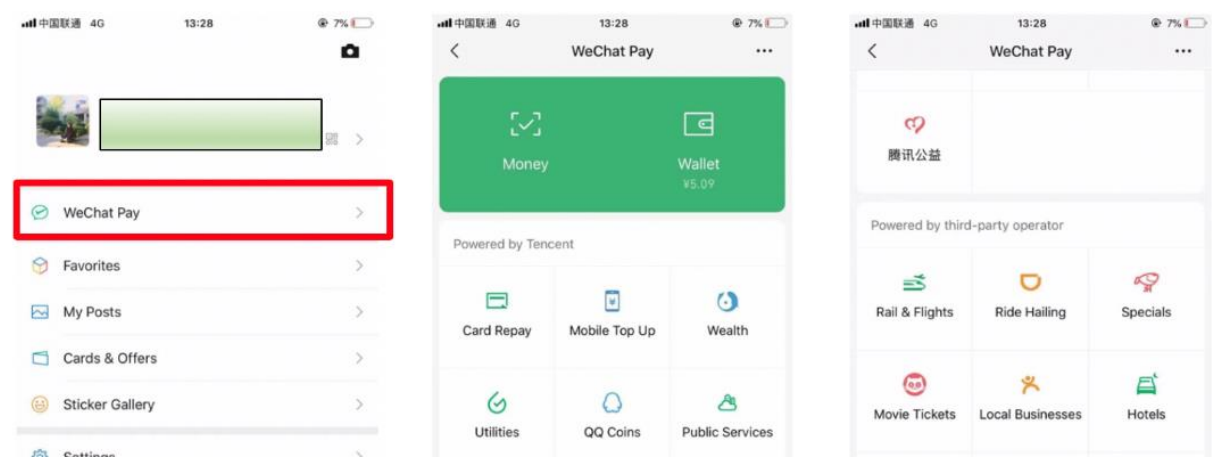

Figure 1. WeChat Pay 


\subsection{Mini Programs}

In 2017, WeChat released a significant new feature called 'mini programs' (see Figure 2); "WeChat mini program is an application embedded in WeChat that users can use without downloading and installing" and thus does not require additional storage space on the smartphone (Chenga et al, 2019: 490). Further, for convenience and ease of use, the mini programs not only incorporate WeChat's default functions, but also additional functions including authentication, payment, sharing and communication (Chenga et al, 2019). Mini programs allow users to engage in various online services, including "paying bills, shopping, finding new friends, getting information, finding stores, reserving taxis, booking appointments, etc" (Wan et al, 2019: 1099).
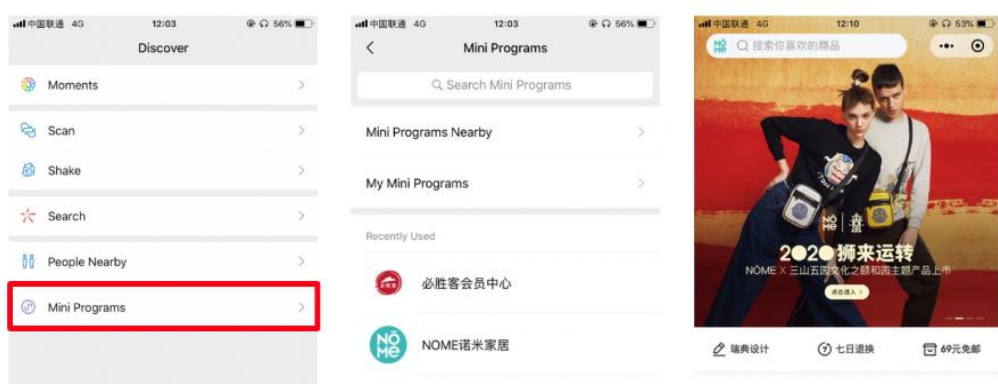

Figure 2. WeChat Mini Programs

\section{COMPARATIVE DISCUSSION}

\subsection{Users}

When beginning to compare WhatsApp and its counterpart WeChat, both applications that started within a period of two years and with similar initial aims and features to provide messaging and calling functionality to users, the first and most noticeable similarity that becomes apparent is their users and popularity. Both applications have gained vast success in terms of their user reach and are the first and third most popular global messenger applications respectively. Moreover, Figure 3 shows the most popular global mobile messenger applications as of October 2019, based on number of monthly active users; the two globally leading applications are WhatsApp with 1.6 billion and Facebook Messenger with 1.3 billion monthly active users. Based on these statistics, WeChat is behind WhatsApp and Facebook Messenger with 1.1 billion.

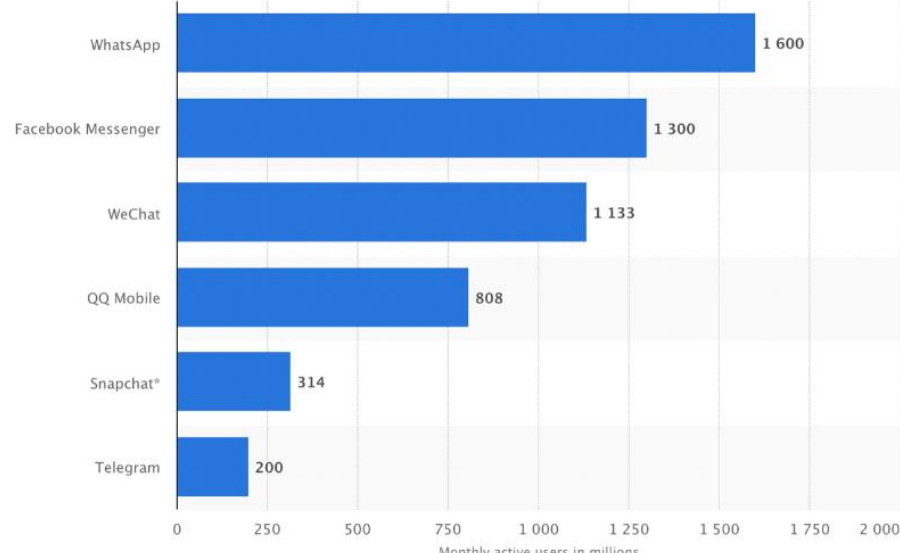

Figure 3. Most popular global mobile messaging apps 2019 (Statista, 2019) 


\subsection{Global Reach}

Whilst in terms of uptake, both applications boast over 1 billion active monthly users, as per Figure 4 that shows the top messaging applications dominating each country, it is clear that WhatsApp has conquered the international market by reaching 180 countries, and in 169 of which it has gained dominance over other messenger applications, whereas WeChat is the leader in China alone (WhatsApp, 2020; MessengerPeople, 2019). Wan et al (2019: 1097) compare the global reach of WhatsApp and WeChat and argue that "WhatsApp succeeded in rapid global expansion through fulfilling consumers' needs for simple, reliable and no- frills functionalities". However, it is important to note that whilst WhatsApp has a wider reach, i.e. its users spread across 180 countries where it is the leading application in 169 of those countries, WeChat has gained its 1.1 billion user-base within a single and more concentrated market.

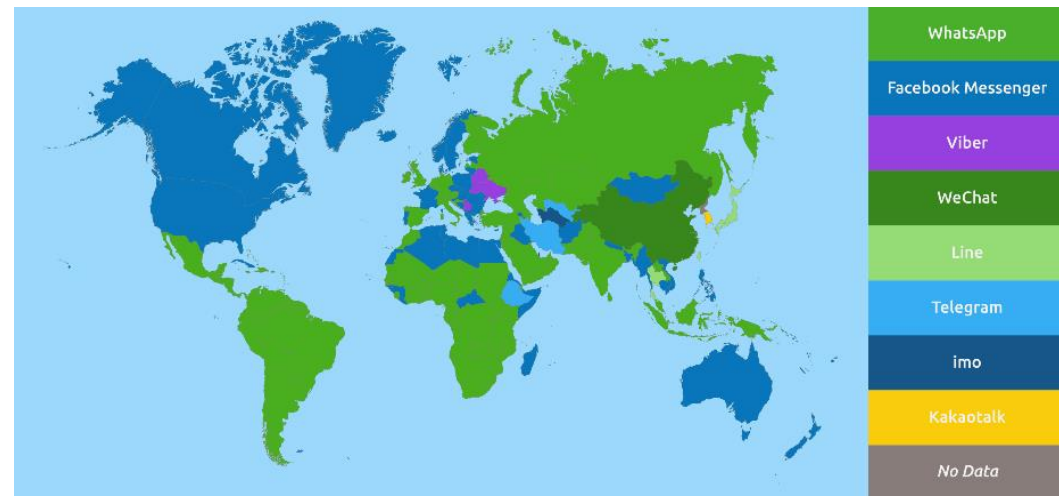

Figure 4. Top messaging apps by country (MessengerPeople, 2019)

\subsection{Functionality}

Table 1. Brief comparison of features between WhatsApp and WeChat

\begin{tabular}{lcc}
\hline Functionality/Feature & WhatsApp & WeChat \\
\hline Cost & Free & Free \\
Founded & 2009 & 2011 \\
Multi-platform & Yes & Yes \\
Security/encryption & End-to-end encryption & Not known \\
Monthly users & 1.6 billion & 1.1 billion \\
Leading in countries & 169 & 1 \\
Text and voice messaging & Yes & Yes \\
Voice and video calling & Yes & Yes \\
Document sharing & Yes & Yes \\
Group chats and calls & Yes & Yes \\
Voice to text & No & Yes \\
Status updates & Yes hours & Yes -3 days - permanent \\
Read receipts & Yes & No \\
Online status & Yes & No \\
Scanning QR codes & No & Yes \\
Mini programs & No & Yes \\
Cashless payment & No & Yes \\
Taxi service & No & Yes \\
\hline
\end{tabular}


When comparing the consumer value proposition (CVP) for WhatsApp and WeChat, Wan et al (2019) state that the biggest differences between the two applications are customers' perceived ease-of-use, perceived usefulness, perceived risk and perceived enjoyment. Furthermore, whilst WeChat's functionality and innovation in its services proceeds WhatsApp in various ways, WhatsApp has succeeded internationally due to its continuous focus on the mass market through its simple yet user-friendly and reliable functionalities; the "benefits that WeChat offers may not necessarily be required/accepted by international consumers due to differences in culture settings and security/privacy risk exposure. WeChat target market is not as universal as WhatsApp" (Wan et al, 2019: 1097). Thus, Montag et al (2018) argue that despite its huge number of users in Asia, WeChat is still not very well-known in Western countries.

Within China, WhatsApp is entirely dependent upon and can only be accessed through the use of a VPN and thus its uptake is limited and restricted, whereas "WeChat is getting more consumers in the West than WhatsApp is in China" (Charlotte et al, 2018: 3). Charlotte et al (2018) also argue that WeChat is not a contestable market due to the security barriers between China and the outside world which are reflected in the permissions and access to technological applications. Also, for many people outside China to communicate with individuals inside China, results in the download of and uptake of WeChat usage. However, WeChat's success is evidently due to deeper reasons than merely the accessibility issues. Whilst one reason for the dominance and prevailing success of WeChat in China could be due to the fact that "social media such as Facebook, YouTube, and Twitter are blocked in the country, WeChat has been specifically created deep-rooted in and based on important Chinese cultural elements" such as collectivism; as culture, cultural values and core beliefs influence our communication patterns, they subsequently play a significant role in the creation of social media platforms (Vodanovich et al, 2017: 618).

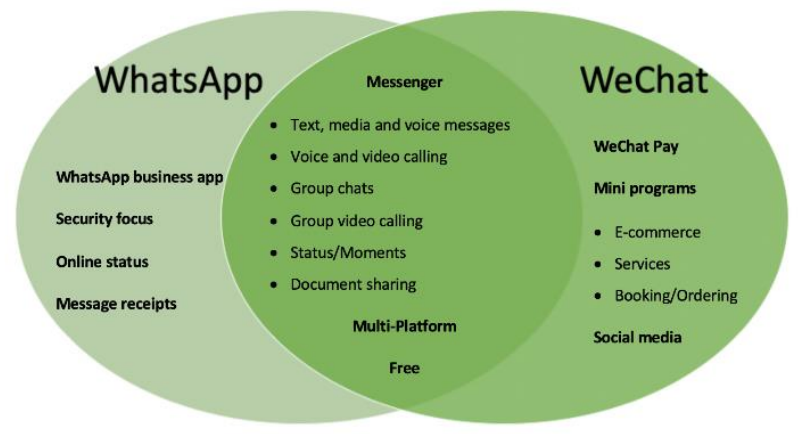

Figure 5. High-level comparison of WhatsApp and WeChat features

\subsection{Security and privacy}

In terms of security, in comparison to WeChat, WhatsApp places a great deal of emphasis on its security features - "Our messages and calls are secured with end-to-end encryption, meaning that no third-party including WhatsApp can read or listen to them. Behind every product decision is our desire to let people communicate anywhere in the world without barriers" (WhatsApp, 2020). Whereas, Wan et al (2019: 1096-1097) claim that "WeChat has a reputation of being heavily monitored" and there are concerns relating to surveillance and privacy. Although such claims are not verified, despite such claims, the success of the application within China has not been hindered but may be a cause behind the lack of international uptake.

From a privacy perspective, WhatsApp shows a user's online activity such as their online status or whether a message has been received and read, which WeChat conceals and thus offers greater user privacy. In addition, for individual users, WeChat only allows communication between contacts, thus in order to communicate with another individual, a contact request must first be sent and accepted by the other user. WhatsApp allows a user to send a message to another WhatsApp user using their telephone number, however, this can be accepted or rejected by the recipient. 


\section{CONCLUSION}

To conclude, despite the setback from the international audiences where WeChat failed to compete with the leading messenger apps such as WhatsApp and take a notable slice of the international market, it has concentrated its primary focus within China. Since its launch, WeChat has become an entire Chinese social media platform that has innovated underpinned by Chinese cultural values (Vodanovich et al, 2017) and demonstrated its success through this dominance; "research has only recently begun to examine the impact of this development on the societal and individual levels" (Montag et al, 2018: 1). On the other hand, whilst WhatsApp's reach is global, its scope and evolution have not changed significantly in the decade since its launch.

WeChat has not only become an integral part of our lives in China and integrated and replaced various other applications and methods for doing things, but has also changed many of our preconceptions about China and its originality, and given an insight and subsequent research focus into the fascinating technological and service innovation within the rich context of the world's most populous country. Thus, the key question we now ask is, can WeChat - that is built on Chinese cultural values - innovate in ways through which it can create a significant influence in the West and succeed globally?

\section{REFERENCES}

Charlotte, H., Solène, R., Coste-Manière, I., Laura, F. and Emmanuelle, M. (2018) WeChat ${ }^{\circledR}$ : The Present and the Future. In IEEE Proceedings of International Conference on Intelligent and Innovative Computing Applications (ICONIC) 2018. Plaine Magnien, Mauritius, 6-7 December, pp. 1-4.

Chenga, A., Renb, G., Hongc, T. and Kood, C. (2019) The WeChat Mini Program for Smart Tourism. Asia Pacific Journal of Information Systems, 29(3), pp. 489-502.

McSweeney, M.A. (2018) The Pragmatics of Text Messaging: Making Meaning in Messages. New York: Routledge.

MessengerPeople (2019) WhatsApp, WeChat and Facebook Messenger Apps - Global Messenger Usage, Penetration and Statistics. [online] Available at: https://www.messengerpeople.com/global-messenger-usage-statistics/ [Accessed 29 December 2019].

Montag, C., Becker, B. and Gan, C. (2018) The Multipurpose Application WeChat: A Review on Recent Research. Frontiers in Psychology, [e-journal] 9(1), p.2247. Available https://www.ncbi.nlm.nih.gov/pmc/articles/PMC6297283/ [Accessed 03 January 2020].

O'Hara, K.P., Massimi, M., Harper, R., Rubens, S. and Morris, J. (2014) Everyday Dwelling with WhatsApp. In Proceedings of the 17th ACM Conference on Computer Supported Cooperative Work \& Social Computing. Baltimore, Maryland, USA, 15-18 February, pp. 1131-1143.

Statista (2019) Most Popular Global Mobile Messenger Apps as of October 2019, based on Number of Monthly Active Users. Available at: https://www.statista.com/statistics/258749/most-popular-global-mobile-messenger-apps/ [Accessed 29 December 2019].

Sulleyman, A. (2018) 'There's a new version of WhatsApp', The Independent, January 19, 2018. Available at: https://www.independent.co.uk/life-style/gadgets-and-tech/news/whatsapp-business-app-download-android-googleplay-iphone-features-a8167631.html/ [Accessed January 2, 2020].

Vodanovich, S., McKenna, B. and Cai, W. (2017) Cultural Values Inherent in the Design of Social Media Platforms: A Case Study of WeChat. . In Proceedings of the 30th Bled eConference. Bled, Slovenia, 18-21 June, pp. 617-628.

Wan, W.S., Dastane, O., Satar, M., Safie, N. and Ma'Arif, M.Y. (2019) What WeChat Can Learn From WhatsApp? Customer Value Proposition Development for Mobile Social Networking (MSN) Apps: A Case Study Approach. Journal of Theoretical and Applied Information Technology, 97(4), pp. 1091-1117.

WeChat (2020) Weixin. [online] Available at: https://weixin.qq.com/ [Accessed 04 January 2020].

WhatsApp (2020) WhatsApp.com. [online] Available at: https://www.whatsapp.com/ [Accessed 05 January 2020]. 